\title{
Sorne theorems on analytic functions in an annulus
}

\author{
By Tadao KuBo \\ (Received August 20, 1959)
}

\section{Preliminaries.}

At the beginning of this paper we introduce some notations which are necessary for the discussion in the sequel.

(i) Let $a$ and $b$ be any two points in the unit circle $|w|<1$ in the $w$-plane, then we denote by $[a, b]$ the hyperbolic distance [7] of these two points, i. e.,

$$
[a, b]=\left|\frac{a-b}{1-\bar{a} b}\right| \text {. }
$$

(ii) We denote the ring-domain bounded by $|w|=1$ and the rectilinear slit $<0,1 / P>(P>1)$ by $G_{P}$ which is called the Grötzsch's extremal domain [1] and whose Riemann modulus is denoted by $\Phi(P)$. It is well-known that $\Phi(P)$ is a strictly increasing function for $P(>1)$. Let $P$ be uniquely determined for a given positive constant $Q$ so as to satisfy the condition

$$
\frac{1}{Q}=\Phi(P)
$$

then the annulus $D: Q<|z|<1$ can be mapped univalently onto $G_{P}$ laid on the $w$-plane by the function $w=F(z ; Q)$ which is called the Grötzsch's extremal function.

(iii) We denote by $\mathfrak{F}$ a class of the functions $w=f(z)$ which are singlevalued and analytic (not necessarily univalent) in the annulus $D$, and whose ranges of values $D_{f}$ for $D$ lie in the circle $|w|<1$ and have the boundary component $|w|=1$ as the image of outer circle $|z|=1$ of $D$.

The object of this paper is to establish some theorems on the ranges of values taken by the analytic functions which are single-valued in $D$ and satisfy various conditions. The proofs are based on the following fundamental theorem [4] which is regarded as an extension of the theorem of Hayman (Theorem II formulated in his paper [2]).

Fundamental THEOREM. Let $e$ be a closed set of real numbers which is contained in the interval $0 \leqq x<1$ and whose Lebesgue measure is at least $1 / P$ $(1 / Q=\Phi(P))$. For each $x \in e$, let be associated a closed set of points $C(x)$ contained entirely in the circle $|w|<1$ such that, if $w_{1}, w_{2}$ be any points on $C\left(x_{1}\right), C\left(x_{2}\right)$ respectively, then the inequality for the hyperbolic distance

$$
\left[w_{1}, w_{2}\right] \geqq\left[x_{1}, x_{2}\right]
$$

always holds. Then the range of values $D_{f}$ taken by a function $w=f(z) \in \mathfrak{F}$ in the annulus $D$ contains at least one of the sets $\{C(x)\}(x \in e)$, except possibly when $e$ is an interval of length $1 / P$ and $w=f(z)$ takes the form

$$
f(z) \equiv S\{F(z ; Q)\} \in \mathfrak{F},
$$

$S$ denoting any linear transformation mapping the unit circular disk $|w|<1$ onto itself. 


\section{Range of values of a function $\in \subseteq$.}

Using the fundamental theorem we shall prove the following

THEOREM 1. Let $\subseteq$ be a class of the functions $\zeta=\varphi(z)$ which are single-valued and analytic (not necessarily univalent) in the annulus $D: Q<|z|<1$, and whose ranges of values $D_{\zeta}$ for $D$ lie in the strip $T: 0<\operatorname{Im} \zeta<1$ and have the boundary component $\operatorname{Im} \zeta=0,1$ as the image of outer circle $|z|=1$ of $D$. Suppose that a function $\zeta=\varphi(z) \in \widetilde{S}$. Then, for each component $E_{j}(j=1,2, \cdots)$ of the complement of $D_{\zeta}$ with respect to $T$, there holds the inequalily

$$
\underset{\zeta \in E_{j}}{\operatorname{Osc}_{j}}(\operatorname{Re} \zeta) \leqq \frac{1}{\pi} \log \frac{P+1}{P-1}, \quad\left(\frac{1}{Q}=\Phi(P)\right) .
$$

The result is best possible.

Proof. Let $\zeta_{0}$ be a point maximizing $\operatorname{Re} \zeta\left(\zeta \in E_{j}\right)$ for a fixed component $E_{j}$ of the complement of $D_{5}$ with respect to $T$. We map the strip $T$ univalently onto the unit circle $|w|<1$ in such a way that the point $\zeta_{0}$ is carried into the origin $w=0$ and the rectilinear segment through the point $\zeta_{0}: \operatorname{Re} \zeta=\operatorname{Re} \zeta_{0}, 0 \leqq \operatorname{Im} \zeta \leqq 1$ into the diameter $\langle-i, i\rangle$ of the circle $|w|=1$. Suppose that the points $w=a\left(=e^{i \theta}\right)$ and $w=b\left(=-e^{-i \theta}\right)(\theta$ : real $)$ correspond to $\zeta=-\infty$ and $\zeta=+\infty$, respectively. Such a univalent mapping is carried out by the inverse function $w=g(\zeta)$ of the function

$$
\zeta=\frac{1}{\pi} \log \frac{w-a}{w-b}+\operatorname{Re} \zeta_{0}+i \mu \quad(\mu \text { : real const.). }
$$

It is clear that the composite function $w=g\{\varphi(z)\} \in \mathfrak{F}$. Denote by $D_{w}$ the range of values taken by $w=g\{\varphi(z)\}$ in $D$.

Nextly, by the function $W=(w-a) /(w-b)$, we transform the right half $\Gamma$ of the system of Apollonius' circles with limit points $a$ and $b$ into the system of concentric circles $|W|=r(0<r \leqq 1)$ in the $W$-plane. We denote by $\Gamma^{\prime}$ the system of circular arcs which lie on the circles belonging to $\Gamma$ and are intercepted by two circular $\operatorname{arcs} K$ and $K^{\prime}$ connecting the points $a$ and $b$ inside the unit circle $|w|=1$. We may take $K$ and $K^{\prime}$ sufficiently near to the circular arcs on $|w|=1: \overparen{a b}$ and $\overparen{b a}$, respectively, such that $K \cup K^{\prime}$ encloses the complement of $D_{w}$.

We now take the interval $0 \leqq x \leqq 1 / P$ for the set $e$ and the circular arc of $\Gamma^{\prime}$ corresponding to $|W|=r$ by a relation

$$
x=\frac{1-r}{1+r}
$$

for each set $C(x)(x \in e)$, and apply the fundamental theorem to the function $w=g\{\varphi(z)\}$. Then the condition (3) for $C(x)$ is satisfied. In fact, if we denote by $W_{j}=r_{j} e^{i \theta_{j}}(j=1,2)$ the point in the $W$-plane corresponding to any point on $w_{j} \in C\left(x_{j}\right)\left(x_{j} \in e, j=1,2\right)$, we obtain the inequality

$$
\begin{aligned}
{\left[w_{1}, w_{2}\right] } & =\left|\frac{w_{1}-w_{2}}{1-\bar{w}_{1} w_{2}}\right|=\left|\frac{W_{1}-W_{2}}{\bar{W}_{1}+e^{-2 i \theta} W_{2}}\right| \\
& \geqq\left|\frac{r_{1}-r_{2}}{r_{1}+r_{2}}\right|=\left|\frac{x_{1}-x_{2}}{1-x_{1} x_{2}}\right|=\left[x_{1}, x_{2}\right],
\end{aligned}
$$

using (7). Hence, from the fundamental theorem, the range of values $D_{w}$ taken by the function $w=g\{\varphi(z)\}$ contains at least one of the sets $\{C(x)\}(x \in\langle 0,1 / P\rangle)$, unless 
the function takes the form $w=S\{F(z ; Q)\}$. Therefore, the range of values $D_{\zeta}$ taken by the function $\zeta=\varphi(z)$ contains at least one of the segments:

$$
\operatorname{Re} \zeta=k\left(\frac{1}{\pi} \log \frac{P-1}{P+1}+\operatorname{Re} \zeta_{0} \leqq k \leqq \operatorname{Re} \zeta_{0}\right), \quad 0<\operatorname{Im} \zeta<1,
$$

except possibly when $\varphi(z) \equiv g^{-1}[S\{F(z ; Q)\}]$. Thus, we have the inequality

$$
\underset{\zeta \in E_{j}}{\operatorname{Osc}}(\operatorname{Re} \zeta)<\frac{1}{\pi} \log \frac{P+1}{P-1} .
$$

Finally, restricting ourselves to the exceptional çase when $\varphi(z) \equiv g^{-1}[S\{F(z ; Q)\}]$, we obtain the result that the equality sign in (5) holds if and only if $\zeta=\varphi(z) \in \mathbb{S}$. maps the annulus $D$ univalently onto the strip $T$ slit along the rectilinear segment: $\operatorname{Im} \zeta=1 / 2$. The details of calculations will be omitted here; cf. [5].

\section{A generalization of Szegö's theorem.}

In 1922, Szegö [6] proved the following theorem:

Let $w=f(z)=z+\sum_{n=2}^{\infty} a_{n} z^{n}$ be regular and univalent in $E:|z|<1$. Let $l$ be the length of any principal chord of the image domain $D_{f}$ for $E$. Then

$$
l \geqq 1 \text {. }
$$

The result is best possible.

In this section we consider the following class $\mathfrak{R}$ of functions: ( $i)$ the functions $w=f(z)$ are single-valued and analytic in an annulus $D: 1<|z|<R$, (ii) the ranges of values $D_{f}$ for $D$ lie in the domain $|w|>1$ and have the boundary component $|w|=1$ as the image of inner circle $|z|=1$ of $D$.

We now attempt to extend the above result to the case of any analytic function $\in \Re$ and prove the following

THEOREM 2. Let $w=f(z) \in \Re$ and let $l$ be the length of any principal chord of the simply-connected domain $\widetilde{D}_{f}$ which is bounded by the outer boundary of $D_{f}$ and contains $D_{f}$. Then there holds the inequality

$$
l \geqq 2\left(P+\sqrt{P^{2}-1}\right),
$$

$P$ being uniquely determined for $R$ by the relation $R=\Phi(P)$. The result is best possible.

Proof. Let $D_{f^{\prime}}$ be the image domain of $D_{f}$ by a transformation $w^{\prime}=1 / w$ and let $E_{j}{ }^{\prime}$ be some component of the complement of $D_{f^{\prime}}$ with respect to the open disk $\left|w^{\prime}\right|<1$. Suppose that $E_{j}^{\prime}$ contains two points $a$ and $-a(a>0)$, lying symmetrically with respect to the origin $w^{\prime}=0$. Then we have

$$
\frac{1}{a} \geqq P+\sqrt{P^{2}-1} \text {. }
$$

In fact, by the univalent function utilized in Sec. $2(a=1, b=-1)$

$$
\zeta=\frac{1}{\pi} \log \frac{w^{\prime}-1}{w^{\prime}+1}-\frac{i}{2}+\lambda \quad(\lambda: \text { real const. })
$$

we map $D_{f^{\prime}}$ onto a subdomain of the strip $T: 0<\operatorname{Im} \zeta<1$, and using Theorem 1 we obtain 


$$
\underset{\zeta \in E_{j^{\prime \prime}}}{\operatorname{Osc}}(\operatorname{Re} \zeta) \leqq \frac{1}{\pi} \log \frac{P+1}{P-1} \quad(R=\Phi(P))
$$

for the image $E_{j}{ }^{\prime \prime}$ of $E_{j}{ }^{\prime}$ by the mapping (12). From the inequality we easily obtain

$$
\frac{1}{\pi}\left(\log \left|\frac{a+1}{a-1}\right|-\log \left|\frac{a-1}{a+1}\right|\right) \leqq \frac{1}{\pi} \log \frac{P+1}{P-1}
$$

and therefore (11)

Returning to the simply-connected domain $\widetilde{D}_{f}$ in the w-plane, let $h_{1}$ and $h_{2}$ be two end-points of any principal chord of $\tilde{D}_{f}$. We may assume that $h_{1}>0$ and $h_{2}<0$ without any loss of generality. Then $l=\left|h_{1}\right|+\left|h_{2}\right|$.

Firstly we consider the case when $\left|h_{1}\right|=\left|h_{2}\right|$. By transforming $\widetilde{D}_{f}$ onto some domain $\widetilde{D}_{f}^{\prime}$ by the mapping $w^{\prime}=1 / w$ and by applying (11), we have

and accordingly

$$
h_{1}=-h_{2} \geqq P+\sqrt{P^{2}-1} \text {, }
$$

$$
l \geqq 2\left(P+\sqrt{P^{2}-1}\right) .
$$

Secondly we consider the case when $\left|h_{1}\right| \neq\left|h_{2}\right|$. Let $\alpha$ be a real constant satisfying the condition

$$
\frac{h_{1}-\alpha}{1-\alpha h_{1}}=-\frac{h_{2}-\alpha}{1-\alpha h_{2}} \text {. }
$$

Such a real constant $\alpha$ can be uniquely determined as the root with smaller absolute value of the quadratic equation

$$
\alpha^{2}-2 \frac{1+h_{1} h_{2}}{h_{1}+h_{2}} \alpha+1=0 .
$$

Then, by a mapping $w^{\prime \prime}=(w-\alpha) /(1-\alpha w)$, the domain $\tilde{D}_{f}$ is transformed onto some domain $D_{f}^{\prime \prime}$ in the $w^{\prime \prime}$-plane whose complement with respect to the domain $\left|w^{\prime \prime}\right|>1$ contains two points $a^{\prime \prime}$ and $-a^{\prime \prime}\left(a^{\prime \prime}=\left|h_{1}-\alpha\right| /\left|1-\alpha h_{1}\right|\right)$, lying symmetrically with respect to the origin $w^{\prime \prime}=0$. Hence there holds

$$
\left|a^{\prime \prime}\right| \geqq P+\sqrt{P^{2}-1}
$$

from the result (11). On the other hand, there holds

$$
\begin{gathered}
\left|a^{\prime \prime}\right|=\left|\frac{h_{1}-\alpha}{1-\alpha h_{1}}\right|=\frac{\left|h_{1}\right| \sqrt{h_{2}^{2}-1}+\left|h_{2}\right| \sqrt{h_{1}^{2}-1}}{\sqrt{h_{1}^{2}-1}+\sqrt{h_{2}^{2}-1}} \\
<\frac{1}{2}\left(\left|h_{1}\right|+\left|h_{2}\right|\right)=\frac{1}{2} l
\end{gathered}
$$

from (14) and an elementary inequality

$$
(a-b)\left(\sqrt{a^{2}-1}-\sqrt{b^{2}-1}\right)>0 \quad(a, b>1 ; a \neq b) .
$$

Thus we also obtain

$$
l>2\left(P+\sqrt{P^{2}-1}\right)
$$

for the case $\left|h_{1}\right| \neq\left|h_{2}\right|$, from (15) and (16). The equality sign never holds for this case.

From the above considerations it is seen that the equality sign of (10) holds only if the image domain of $D$ in the $\zeta$-plane by a mapping

$$
\zeta=\frac{1}{\pi} \log \frac{1 / w-1}{1 / w+1}-\frac{i}{2}+\lambda \quad(w=f(z) \in \Re)
$$


is schlicht and coincides with the strip $T$ slit along the segment $\operatorname{Im} \zeta=1 / 2$. Thus there occurs the equality if and only if the function $w=f(z) \in \Re$ univalently maps the annulus $D$ onto the domain $|w|>1$ slit along the segment on the real axis: $|w| \geqq P+\sqrt{P^{2}-1}$, up to a rotation about the origin $w=0$. Therefore the inequality (10) is best possible.

REMARK. Performing the transformations $Z=z / R$ and $W=w / R$ to the domains $D$ and $D_{f}$ respectively, we obtain Szegö's theorem from Theorem 2 as the limiting case when $R \rightarrow \infty$, because the right-hand side of (10) divided by $R$ tends to 1 [3].

Kyoto Prefectural University of Medicine.

\section{References}

[1] Grötzsch, H., Über einige Extremalprobleme der konformen Abbildung, I; II. Leipziger Ber. 80 (1928), 367-376; 497-502.

[2] Hayman, W. K., Some applications of the transfinite diameter to the theory of functions. J. Anal. Math. 1 (1951), 155-179.

[3] Komatu, Y., Untersuchungen über konforme Abbildung von zweifach zusammenhängenden Gebieten. Proc. Phys.-Math. Soc. Japan 25 (1943), 1-42.

[4] Kubo, T., Hyperbolic transfinite diameter and some theorems on analytic functions in an annulus. J. Math. Soc. Japan 10 (1958), 348-364.

[5] - On a theorem of analytic functions in an annulus. Math Japonicae 5 (1958), 17-20.

[6] Szegö, G., Aufgabe 2 und ihre Lösung. Jbr. Deut. Math.-Verein. 31 (1922), p. $42 ; 32$ (1923), p. 45.

[7] Tsuji, M., Potential theory in modern function theory. Tokyo, 1959. 